\title{
La mise en réseau de ressources en soutien aux entreprises
}

\author{
Judith Bergeron ${ }^{1}$ \\ Conseil national de recherches Canada
}

\section{Introduction}

Au moment de définir ce qu'est un réseau, les questions qui viendront rapidement seront de l'ordre du : " qui composent le Réseau », « quels sont les critères pour les sélectionner », "que font-ils ensemble ». Prenons d'abord le temps de revoir quelques points qui définissent la nature d'un réseau. Ensuite, nous pourrons déterminer ce que la nature de réseau peut dicter comme type d'objectifs à atteindre pour enfin donner quelques recommandations sur la façon de mesurer les résultats d'un réseau.

\section{Un réseau : une affaire de personnes et de confiance}

En premier lieu, il faut rappeler un constat fondamental au moment de parler des réseaux : c'est avant tout une affaire de personnes, tout comme l'entrepreneuriat, pour faire écho à la présentation de Nathalie Riverin, donnée lors de la journée « Mieux épauler l'entrepreneur, notre défi : sa réussite $»$.

Plus encore, les réseaux sont faits de la confiance que les personnes qui les composent ont les unes envers les autres. Il faut ajouter que la confiance que les gens ont dans les autres est le reflet, entre autres, de la confiance qu'ils ont en eux, en leurs capacités et dans l'environnement dans lequel ils évoluent.

\section{Créer un climat où les gens sont en sécurité}

Il y a donc des pré-requis à la mise en place d'un « réseau », si ce n'est que celui de créer un climat ou les personnes invitées à former un «réseau » auront confiance que le cadre dans lequel se fait cette invitation est " sécuritaire ». La perception de sécurité sera différente pour chacun, mais certains éléments peuvent être communs à tous : l'assurance qu'il n'y aura pas perte d'emploi ou perte de ressources financières pour réaliser des projets, par exemple. Dans un réseau de professionnels, la crainte de devoir éventuellement changer sa façon d'intervenir, alors qu'elle remporte de grands succès, peut être perçue comme une perte importante. Les pratiques liées à la gestion du changement sont utiles dans ce contexte.

\section{Un réseau est « organique »}

Comme les réseaux sont le fait de la confiance entre les personnes, il va de soi que leur développement ne peut être brusqué. Peu de temps après avoir commencé mon rôle d'animatrice de réseau, j'ai constaté qu'un réseau a une vie à lui. J'ai commencé à expliquer qu'il « respirait». Quelquefois, plusieurs personnes y arrivaient, à d'autres moments, le groupe central était plus petit. Un réseau et son évolution sont « organiques » et tout comme on ne peut pas tirer sur une fleur pour qu'elle pousse, le développement d'un réseau demande donc de la patience et beaucoup de soins. Si on tire trop sur la fleur, on l'arrache. On peut traduire ce principe par des mots beaucoup plus concrets : les réseaux sont le fait de contributions volontaires, les obligations sont tota- 
lement inefficaces et vont même nuire au développement et au dynamisme des réseaux. Un réseau a donc des étapes et des types d'activités qui marquent sa maturité grandissante. À l'exception de la première activité mentionnée, les autres s'accumulent dans le temps et doivent aussi se renouveler tout en gagnant en profondeur.

Tableau 1

Activités marquant l'évolution de la maturité d'un réseau

\begin{tabular}{|c|c|}
\hline \multicolumn{1}{|c|}{ Étapes } & Activités \\
\hline Mobiliser des premiers individus autour d'une mission et d'une vision à créer & Vision, conception \\
\hline $\begin{array}{c}\text { Mobiliser des premiers individus autour de cette mission et des objectifs qui en } \\
\text { découlent }\end{array}$ & Recrutement \\
\hline Faire exister le réseau & Activités, rencontres \\
\hline Donner de la valeur ajoutée pour ceux qui composent le réseau & Outils, services \\
\hline Écouter les gens qui composent le réseau & Ateliers \\
\hline Mettre les gens en action réseau, les responsabiliser & Promotion \\
\hline Documenter les retombées & Reconnaissance \\
\hline
\end{tabular}

\section{Le parrain}

Comme les réseaux doivent grandir à leur propre rythme, c'est pour ça que ceux qui réussissent ont tous un parrain, un organisme (et un ou des individus au sein de cet organisme) qui, à travers tous les aléas des incertitudes économiques, politiques, sociales, maintiendra la vision, et soutiendra une infrastructure de base pour animer et favoriser le développement du réseau. Le parrain doit soutenir le réseau de façon altruiste. Ce réseau n'est pas à son service, mais un moyen supplémentaire pour générer des retombées pour une partie ou la totalité de sa clientèle, mais sans que ces retombées lui soient directement attribuées.

\section{La réciprocité et la complémentarité : des valeurs déterminantes}

Les personnes faisant partie d'un réseau à succès véhiculent et vivent des valeurs qui en favorisent le développement. Une première valeur intrinsèque à tout réseau est celle de la réciprocité. Cette valeur se traduit par le fait que ceux et celles qui y adhèrent ont la conviction que lorsque l'on donne, on reçoit. Ceux et celles qui auront un effet bénéfique sur le développement d'un réseau seront les personnes qui y mettront du temps, qui y prêteront leurs talents, leurs idées, leurs visions. Ils seront généreux, car ils seront convaincus, pour l'avoir déjà expérimenté, que leurs efforts leur rapporteront, à cause du principe de réciprocité. Ces personnes seront les précurseurs, elles seront celles autour de qui la dynamique du réseau s'organisera. Elles ne chercheront pas à garder pour elles ni les idées, ni la reconnaissance; elles voudront les donner au réseau, à la communauté.

La deuxième sera certainement celle de la complémentarité. En effet, mettre l'accent sur les connaissances, les expériences et les succès variés des différentes personnes du réseau favorisera les rapprochements dans un contexte d'enrichissement plutôt que de concurrence. Cela voudra dire qu'au moment de donner de la visibilité à certaines pratiques à succès, ces opérations doivent viser à inspirer les participants et non laisser planer le doute que cette pratique sera imposée dans le futur. Inviter les gens qui ont envie de partager leurs bons coups sera une façon de développer la dynamique du réseau par delà les investissements des précurseurs.

Les gens adhéreront à un ou des réseaux seulement lorsqu'ils percevront qu'ils perdront plus à en être exclus qu'à y être inclus. Pour quelques individus le sentiment de sécurité tient à la capacité de "posséder» de façon exclusive incluant le titre de «meilleur service conseil », par exemple. Ces personnes auront de la difficulté à adhérer à une dynamique réseau (d'échange et de partage). Voilà pourquoi il 
est important de maintenir une culture de contribution volontaire pour développer un réseau sain. Il est préférable que ceux et celles qui ne se sentent pas à l'aise dans la dynamique réseau ne soient pas obligés d'y adhérer.

\section{Une fonction d'animation dédiée}

Pour qu'un réseau gagne plus rapidement en maturité, une fonction d'animation dédiée sera un facteur de succès important à considérer. Bien sûr, la ou les personnes responsables de cette fonction devront adhérer naturellement aux valeurs de réciprocité et de complémentarité afin de favoriser les échanges et la circulation du savoir et des connaissances dans le réseau. Des profils en communication doteront cette fonction d'une curiosité qui profitera au réseau au moment d'organiser la connaissance et la faire circuler tout en lui assurant des outils de qualité et des contenus pertinents pour les publics cibles.

Des habiletés d'animation seront également un atout afin de pouvoir faire interagir des groupes de personnes ensemble et leur prodiguer des expériences d'équipe et de groupe constructives qui développeront le sentiment d'appartenance à cette communauté qui gagnera en maturité grâce à leur propre engagement.

Il est important de souligner que la ou les personnes dédiées à cette fonction doivent être clairement au service de la mission et des objectifs du réseau, soient ceux qui mobilisent les membres du Réseau. Elles véhiculent, en parole et en action, la mission, la vision, les valeurs et les objectifs du réseau.

\section{Le plaisir}

C'est la première fois que je mentionne le plaisir comme un ingrédient incontournable du succès d'un réseau... probablement parce que ça pourrait ne pas faire assez sérieux... Et pourtant, il est au centre de la vitalité d'un réseau : le plaisir de se rencontrer, le plaisir d'apprendre, le plaisir de se trouver des points communs ou celui de se découvrir des talents complémentaires, le plaisir de partager sa passion avec des gens qui sont curieux de la découvrir ou celui de comprendre celle d'un collègue pour mieux en faire profiter un client, le plaisir de faire mieux...
Le plaisir est indispensable parce que, rappelonsnous, le développement d'un réseau s'appuie sur les contributions volontaires : peu de gens vont réaliser des tâches que ne leur apportent pas de plaisir lorsqu'ils n'y sont pas obligés!

\section{Une mission et des objectifs communs mo- bilisateurs}

Une mission qui mobilise les individus d'un réseau est alignée sur les missions des organisations où oeuvrent les individus qui forment le réseau. Il y aura donc un recoupement important entre la mission du réseau formé et celle des organisations. Ceux qui choisiront de le voir de façon inclusive y adhéreront, y verront un moyen de faire mieux pour leurs clients avec les ressources existantes. Ceux qui choisiront de voir le réseau de façon exclusive, y verront une menace. Cette façon de voir les choses est plus souvent le propre de la direction d'une organisation plutôt que celle des professionnels sur le terrain. Il est donc possible de voir des individus adhérer à l'approche réseau alors que leur organisation ne l'a pas fait. Le réseau doit avoir la souplesse de pouvoir accueillir ces gens de façon informelle avec le temps.

Une mission et des objectifs communs seront mobilisateurs s'ils sont conséquents avec ceux du professionnel et de son organisation. En effet, comme le réseau s'appuie sur les contributions volontaires des individus, les contributions seront plus nombreuses si l'individu peut, à la fois, rencontrer les « objectifs réseau » et rencontrer ses propres objectifs personnels avec plus d'efficience et d'efficacité. Inversement, le réseau aura plus de chance de générer des retombées réseau si sa mission et ses objectifs sont alignés sur ceux de ses organisations membres et des individus qu'il souhaite actifs dans le réseau.

Une mission et des «objectifs réseau » mobilisateurs seront tels qu'ils ne pourront être réalisés par une personne seule. Ainsi, le partage et la collaboration entre professionnels seront nécessaires pour y arriver. Plus encore, ces objectifs devront projeter les individus à l'extérieur du réseau. Autrement dit, les objectifs devront amener les individus à travailler ensemble mais pour le bien d'un client externe. Sinon, les gens du réseau sont tournés l'un vers 
l'autre dans une boucle fermée sans direction sur ce qu'ils ont à accomplir ensemble. Les objectifs doivent les amener à se dépasser auprès d'un client externe. Pour un réseau de professionnels qui conseillent des PME, il est tout indiquer que la mission et l'objectif soient tournés vers le souci de mieux desservir cette clientèle.

\section{Un réseau pourquoi?}

Maintenant que nous avons précisé la nature d'un réseau sain et efficace, nous sommes mieux outillés pour se poser une première question d'importance. Pourquoi mettre un réseau en place? En effet, quand nous comprenons la nature d'un réseau, nous pouvons valider quels types d'objectifs ce moyen permet de rencontrer, ou non.

\section{Objectifs administratifs}

L'idée de la mise en réseau peut être lancée par les partenaires financiers. Quels sont leurs besoins? Cherchent-ils une façon de rationnaliser leurs investissements? Cherchent-ils une lecture plus standardisée des résultats obtenus, ou plus simplement, cherchent-ils une lecture plus significative des résultats pour mieux défendre leur dossier? Veulentils centraliser leurs investissements pour rationaliser le temps de gestion de leurs ententes de financement? Tous ces besoins sont légitimes. Toutefois, la mise en place d'un réseau n'est peut-être pas la meilleure solution. Ces objectifs, qui sont associés à des dynamiques de contrôle et d'obligations risquent même de faire une très mauvaise presse à l'approche réseau, qui pourrait ainsi être «brûlée ». Ces objectifs relèvent de la nature de la gestion des ententes de financement et de la «relation d'affaires » entre le financier et l'organisme financé.

\section{Objectifs d'amélioration, d'innovation dans les services}

D'un autre côté, la mise en place d'un réseau relève d'une approche volontaire où les gens du réseau choisissent de contribuer, partager, échanger pour faire mieux ensemble. Les objectifs naturels pour un réseau seront donc en lien avec ce que les gens peuvent mieux faire ensemble pour rencontrer leurs objectifs existants. Nous sommes dans les sphères du développement professionnel, de l'amélioration et de l'innovation dans les services. Nous sommes dans le domaine de l'investissement dans les ressources et non pas dans celui de la rationalisation. Ici, les indices de performance seront rattachés, par exemple, à ce que les gens auront appris (existence de ressources spécialisées, une réalité émergente dont doit tenir compte l'entrepreneur, etc) où à ce que les gens réaliseront différemment (références à des collègues du réseau, travail en équipe, etc.) Ici, nous sommes dans le domaine de la création et de la connaissance.

Un réseau pourra donc être mis sur pied, par exemple, pour enrichir les services offerts aux PME. Par contre, il ne serait pas recommandé de lui donner l'objectif d'offrir des services standardisés. Voici un tableau qui reprend certains éléments qui reflètent la nature d'un réseau et comment des actions peuvent en favoriser le développement et de rayonnement ou, au contraire, lui nuire.

Tableau 2

\section{Éléments déterminants d'un réseau et les actions associées à ces éléments pouvant être favorables ou nuisibles au développement du réseau}

\begin{tabular}{|l|l|l|}
\hline $\begin{array}{c}\text { Nature d'un réseau : élé- } \\
\text { ments déterminants }\end{array}$ & \multicolumn{1}{|c|}{ Actions à succès } & \multicolumn{1}{c|}{ Actions nuisibles } \\
\hline Développement organique & $\begin{array}{l}\text { Suggérer l'adhésion au réseau, laisser } \\
\text { venir. }\end{array}$ & $\begin{array}{l}\text { Obliger les organisations ou les indivi- } \\
\text { dus à s'engager }\end{array}$ \\
\hline Base volontaire & $\begin{array}{l}\text { Inspirer les gens à participer pour se } \\
\text { dépasser, pour inventer. }\end{array}$ & $\begin{array}{l}\text { Dicter comment agir, contrôler les } \\
\text { actions. }\end{array}$ \\
\hline Parrain & $\begin{array}{l}\text { Contribution désintéressée pour le bien } \\
\text { du client externe du réseau. Le réseau } \\
\text { appartient à tous ses membres. }\end{array}$ & $\begin{array}{l}\text { Avoir un parrain qui agit comme « pro- } \\
\text { priétaire » du réseau qu'il met à son } \\
\text { service. }\end{array}$ \\
\hline Personnes & $\begin{array}{l}\text { Personnalisé tous les aspects de } \\
\text { l'animation du réseau pour développer }\end{array}$ & $\begin{array}{l}\text { Automatiser les actions et les commu- } \\
\text { nications en croyant que les outils in- }\end{array}$ \\
\hline
\end{tabular}




\begin{tabular}{|c|c|c|}
\hline Nature d'un réseau : élé- & Actions à succès & Actions nuisibles \\
\hline & $\begin{array}{l}\text { toujours plus le sentiment } \\
\text { d'appartenance. Mettre les outils in- } \\
\text { formatiques au service de cette appro- } \\
\text { che. }\end{array}$ & $\begin{array}{l}\text { formatiques créent par eux-mêmes une } \\
\text { dynamique réseau. }\end{array}$ \\
\hline Individu & $\begin{array}{l}\text { Trouver et brancher LA personne qui } \\
\text { croit au réseau. (autant chez les parte- } \\
\text { naires financiers que chez les org. } \\
\text { membres) }\end{array}$ & $\begin{array}{l}\text { Imposer le réseau à des personnes à } \\
\text { cause de leur titre (autant chez les par- } \\
\text { tenaires financiers que chez les org. } \\
\text { membres) }\end{array}$ \\
\hline $\begin{array}{l}\text { Au service d'un client } \\
\text { commun, externe }\end{array}$ & $\begin{array}{l}\text { Tous les membres et professionnels } \\
\text { sont au service du client externe. }\end{array}$ & $\begin{array}{l}\text { Certains membres cherchent à mettre le } \\
\text { réseau au service de leur propre intérêt. }\end{array}$ \\
\hline $\begin{array}{l}\text { Mission et objectifs com- } \\
\text { muns }\end{array}$ & $\begin{array}{l}\text { Les objectifs sont en lien avec la clien- } \\
\text { tèle externe tout en étant en lien avec } \\
\text { les missions et les objectifs des organi- } \\
\text { sations membres et des partenaires } \\
\text { financiers face à cette clientèle. }\end{array}$ & $\begin{array}{l}\text { La mission et les objectifs servent les } \\
\text { intérêts de quelques organisations. }\end{array}$ \\
\hline $\begin{array}{l}\text { Objectif tourné } \quad \text { vers } \\
\text { l'extérieur }\end{array}$ & $\begin{array}{l}\text { Se concentrer sur des services qui amé- } \\
\text { liorent le service aux PME }\end{array}$ & $\begin{array}{l}\text { Se concentrer sur les besoins du finan- } \\
\text { cier et sur ceux de quelques organisa- } \\
\text { tions. }\end{array}$ \\
\hline Service amélioré & $\begin{array}{l}\text { Viser et entretenir la diversité et la } \\
\text { complémentarité des expériences, des } \\
\text { connaissances, des compétences en le } \\
\text { mettant au service d'une offre de ser- } \\
\text { vice. L'harmoniser grâce à l'approche } \\
\text { réseau (toutes les entreprises peuvent } \\
\text { recevoir des conseils en «...» parce } \\
\text { qu'un des centres dispose de cette res- } \\
\text { source et la rend disponible partout elle } \\
\text { est en demande. }\end{array}$ & $\begin{array}{l}\text { Viser des services standardisés en ren- } \\
\text { dant tous les intervenants et toutes les } \\
\text { interventions semblables. }\end{array}$ \\
\hline Réciprocité & $\begin{array}{l}\text { Enseigner aux gens que les personnes } \\
\text { doivent venir au réseau pour donner, } \\
\text { s'engager et faire du réseau leur réseau. }\end{array}$ & $\begin{array}{l}\text { Agir comme si les gens se joignent au } \\
\text { réseau pour recevoir. Ne pas les res- } \\
\text { ponsabiliser par rapport au succès du } \\
\text { réseau. }\end{array}$ \\
\hline Complémentarité & $\begin{array}{l}\text { Enseigner aux gens qu'ils doivent venir } \\
\text { au réseau avec l'intention de découvrir } \\
\text { les talents des autres. }\end{array}$ & $\begin{array}{l}\text { Développer une culture où les person- } \\
\text { nes valorisées sont celles qui savent } \\
\text { tout, qui n'ont besoin de personne et } \\
\text { qui ne demandent pas d'aide. }\end{array}$ \\
\hline Reconnaissance des succès & $\begin{array}{l}\text { Reconnaître les actions réseaux souhai- } \\
\text { tées }\end{array}$ & $\begin{array}{l}\text { Ne pas reconnaître ses actions ou en- } \\
\text { core, reconnaître des comportements } \\
\text { non-réseau. }\end{array}$ \\
\hline
\end{tabular}

\section{Un réseau pour qui?}

Je préfère poser cette question avant de demander qui composera le réseau. En effet, les gens qui composent le réseau doivent développer un réseau qui est au service d'un client externe. Il sera important de décrire ce client. Cela peut paraître trivial, mais pour les organismes financés par de l'argent public, cet exercice est toujours périlleux : comment dire non alors que le gouvernement est au service de tous...
Par contre, dans le cadre de la mise sur pied d'un réseau, on peut profiter de l'occasion pour prioriser ou privilégier un type de clientèle qui a, par exemple, des besoins plus complexes. Ainsi, pour ces clients plus spécifiques, les membres travailleront peut-être plus naturellement ensemble puisque, seuls, ils auront rarement toutes les compétences, services et réseaux de contacts pour venir à bout de tous ses besoins. Il sera très important ici que la vision de départ soit très claire et qu'elle soit toujours celle d'un client commun, même si elle se développera sur plusieurs années au sein du réseau. 
Elle ne doit jamais être perdue de vue. Plusieurs petites décisions favoriseront l'émergence de cette notion avec les années, comme celles liées à la mesure de la performance dont nous parlerons plus loin.

\section{Un réseau formé « de qui »?}

Une fois le réseau bien branché sur son client externe, du coup, il est beaucoup plus facile de décider des personnes qui devront le composer. Si on parle de bien servir le client, alors on parlera de gens qui sont en contact direct avec ce client. C'est aussi en discutant des besoins de ce «client commun avec besoins complexes » que nous pourrons préciser davantage le type de personnes qui composeront le réseau.

\section{On obtient ce que l'on mesure}

Il est extrêmement important de bien choisir ce que l'on mesure puisque, on le sait, on obtient ce que l'on mesure. Voilà pourquoi il faut doter les membres du réseau d'objectifs externes qui éviteront une dynamique où les gens qui le composent sont tournés vers eux-mêmes et ont tout le loisir de se voir comme concurrents puisqu'ils se définissent alors un par rapport à l'autre. S'ils se définissent par rapport à leur «client commun avec besoins complexes », ils auront plus de latitude pour observer combien et comment ils sont complémentaires. Du coup, nous aurons aussi plus de chance d'avoir des retombées chez les PME!

S'ils se définissent par rapport à ce qu'ils ont appris ensemble, là encore, nous sommes dans une dynamique projetée vers l'avenir, vers l'avant, vers l'acquisition de nouvelles capacités. Ici, nous générerons de nouvelles connaissances et un souci toujours plus grand de service à valeur ajoutée.

Rappelons-nous qu'un réseau est performant dans un contexte d'acquisition de connaissances et d'amélioration des services et non pas dans un contexte administratif. En m'inspirant de ma propre expérience en animation de réseau de soutien aux entreprises, les indicateurs de performance pourraient être liés à ces aspects :
- Ce qui a été appris (et qui peut aider un client)

- Les personnes qui auront été rencontrées (et qui peuvent aider un client)

- Comment le client a été aidé autrement, mieux, etc.

- Comment le client a été aiguillé vers une autre ressource

\section{Un réseau est performant dans un contexte d'acquisition de connaissances et \\ d'amélioration des services et non pas dans un contexte administratif.}

Mieux vaut avoir peu d'indicateurs de performance réseau (rappelons-nous que les personnes y contribuent sur une base volontaire), mais que ces indicateurs soient vraiment significatifs pour la gestion du réseau. Par exemple, si l'objectif est d'enrichir le service à la PME par leur mise en réseau, il sera très pertinent de mesurer le nombre de contacts donnés aux clients. Évidemment, le client ainsi aiguillé « en réseau » devra être attribué à tous ceux et celles qui auront contribué à sa progression dans le réseau pour que la notion de concurrence soit éliminée de l'équation et que la culture réseau soit reconnue.

Il ne faut jamais oublier de se questionner sur l'effet pernicieux des indicateurs de performance et surtout sur les objectifs quantitatifs qui en découlent. Qu'arriverait-il si les personnes réalisaient les actions seulement pour rencontrer leurs objectifs alors qu'il n'y aucune réelle valeur ajoutée à avoir posé les actions? Dans le cas des exemples de référence, il n'y aurait aucune valeur perçue par les collaborateurs, ni par le client. L'initiative réseau pourrait alors être perçue comme inutile, voire encombrante puisqu'elle demande plus d'efforts sans pour autant générer des retombées ou une satisfaction plus grande. Ce risque est important à considérer au moment de réfléchir à la mesure de rendement.

Bien que ce ne soit pas dans les habitudes des partenaires financiers de soutenir un financement sans objectifs annoncés, l'espace réseau doit d'abord émerger et les mesures de rendement devraient émaner de ce que le réseau lui-même génère avec le temps. N'oublions pas que les «actions réseau » sont réalisées sur une base volontaire et que les «objectifs réseau » doivent émaner du réseau lui- 
même et ne pas être imposés : les obligations tuent un réseau!

Aussi, comme les actions réseau réalisées par les professionnels le sont sur une base volontaire, il y a toujours un défi à les documenter, à moins que les organisations membres intègrent cette mesure à leur propre mode de mesure de rendement et partagent ces résultats, sur base volontaire aussi, avec les personnes responsables de la coordination du réseau.

\section{Un terrain fertile}

Depuis les 2 dernières années environ, je perçois un mouvement naturel de la part des organismes de développement économique de "première ligne" vers cette nouvelle façon de travailler et de concevoir l'intervention en entreprise. En effet, quelques organismes répondant à ces critères se sont joints au RCT au cours de cette dernière année, intéressés par une mise en réseau avec des organismes et des services plus pointus pour soutenir l'innovation en PME. Le contexte me semble donc propice à encourager ce type d'initiatives qui s'inscrivent dans les tendances lourdes de soutien aux entreprises.

\section{La mise en réseau réussie est celle qui est faite sur une base volontaire et dans un climat de confiance. Conclusion}

Les réseaux sont un moyen bien adapté à l'innovation, à la création et à l'amélioration des services mais ne sont pas indiqués pour standardiser ou encore contrôler l'offre d'un groupe d'organisations. La mise en réseau réussie est celle qui est faite sur une base volontaire et dans un climat de confiance. Elle s'appuie sur une mission et des objectifs tournés vers l'extérieur du réseau et sur des valeurs de réciprocité et de complémentarité.

Un réseau se construit et gagne en maturité dans le temps. La mise en réseau doit ajouter de la valeur aux gens qui le composent et aux clients qu'ils desservent ensemble. Les activités qu'ont y retrouvent s'enrichissent et se renouvellent avec le temps. Ces activités ont avantage a être confiées à des personnes qui seront dédiées à l'animation du réseau si on souhaite que le réseau gagne en maturité plus rapidement.

Les mesures de rendements devront être adaptées à la réalité organique du développement de réseau et devront prendre en compte que les personnes contribuent au réseau sur une base volontaire.

Enfin, il est important de voir la mise sur pied dans un contexte de développement et d'investissement et non de rationalisation des effectifs. Déjà, cette donnée est nécessaire pour sécuriser les personnes qui sont invitées à être partie prenante du projet. Mais au-delà de cette réalité, la mise sur pied d'un réseau suppose des investissements dans le temps pour, par exemple :

- Le salaire de la ou des personnes dédiées à l'animation

- Les frais rattachés à l'organisation d'activités (professionnels, par exemple)

- Les frais rattachés à des services de professionnels pour certaines réalisations (à titre d'exemple, réalisation d'outils de communication... puisque le sujet de visibilité est souvent un enjeu pour les membres d'un réseau)

- Les frais rattachés à des services pour les membres afin de soutenir leurs actions réseau (site où repérer les professionnels du réseau par compétences, par exemple).

\section{Notes et références}

1

Depuis la rédaction de ce texte, Judith Bergeron s'est joint au Groupement des chefs d'entreprise du Québec, un réseau d'entrepreneurs qui progressent par l'entraide et l'échange d'expérience. 


\section{Publicité}

«CEE » 